\title{
Mesenchymal Stem Cell Therapy-Is the Vessel Half Full or Half Empty?
}

\author{
Yu. V. Sukhanov ${ }^{a, *}$, E. A. Vorotelyak ${ }^{a}$, I. V. Lyadova ${ }^{a}$, and A. V. Vasiliev ${ }^{a}$ \\ ${ }^{a}$ Koltzov Institute of Development Biology, Russian Academy of Sciences, Moscow, 119334 Russia \\ *e-mail: yuri.sukhanov@gmail.com
}

Received April 28, 2020; revised April 30, 2020; accepted May 2, 2020

\begin{abstract}
The urgency of the search and introduction into medical practice of the method for the therapy of severe forms of pneumonia COVID-19 is due to the lack of effective treatment methods that can destroy the pathogen. Expectations of a good clinical effect from the application of mesenchymal stem cells (MSCs) are not groundless: there is a scientific justification in using MSCs for the treatment of inflammatory diseases and of the proven mechanisms of their action. Along with this, there are very little reliable data about the mechanism of MSCs' action when they are systemically administrated to a human or on the distribution of cells in the body and the long-term consequences of such administration. Data from model experiments are contradictory both concerning the specific action of MSCs and their safety. If clinical studies show an acceptable risk/benefit ratio for the application of MSCs, countries in which such studies have been conducted can expect their introduction into medical practice. In Russia, it is necessary to initiate experimental verification of the specific action of MSCs and the risks of their use in COVID-19 conditions in a sufficient quantity, and, in parallel, to create a mechanism for accelerated but justified admission of biomedical cell products into practice.
\end{abstract}

Keywords: mesenchymal stem cells, MSCs, SARS-CoV-2, pneumonia, COVID-19, inflammation, cytokines, clinical trials, biomedical cell products

DOI: $10.1134 / \mathrm{S} 1062360420040104$

Among the many problems of cell technologies, the most relevant issue is the clinical use of mesenchymal stem cells (MSCs). These cells attract attention as the most accessible resource for cell technologies and suitable for solving many medical problems. Longterm efforts to transdifferentiate MSCs into other types of cells, including neural, hematopoietic, as well as into hepatocytes, cardiomyocytes, and pancreatic cells, etc., have not led to the expected results, but attempts to use MSCs as a systemic regulator of inflammatory and reparative processes continue.

In this regard, attempts to use MSCs for the treatment of patients with severe SARS-CoV-2 forms were not unexpected. The necessity of an urgent search into a method for the therapy of the most severe forms of pneumonia COVID-19 and its introduction into medical practice is caused by the lack of targeted therapies that destroy the pathogen and also by specific features of patients with the highest risk of death and of the patients of the older age group with complications of other diseases. We should also not forget about the commercial motivation of the desire to introduce MSCs into clinical practice.

Expectations to get a clinical effect from the application of MSCs are not groundless: there is a scientific justification for the use of MSCs in anti-inflammatory therapy, including proven mechanisms of their action.

One of the main characteristics of MSCs is their immunomodulatory/immunoregulatory activity, which is provided both by intercellular contacts and paracrynally due to the expression and secretion of a large number of molecules with immunomodulatory properties by MSCs (Lyadova, et al., 2016; Jiang and Xu, 2020). These molecules include the cytokines TGF- $\beta$, IL-10, enzymes indolamine-2,3-dioxygenase (IDO), arginase-1 (Arg1), inducible NO-synthase (NOS2), prostaglandin E2 (PGE2), nonclassical molecules of the main histocompatibility complex type 1 (HLA-G2), CD39 and CD73 molecules (expressed on MSCs, jointly provide the cleavage of ATP to adenosine and have immunosuppressive activity), and galectins (Gal-1 and Gal-9). These factors inhibit the proliferation of T-lymphocytes and NK cells, the formation and activity of type 1 T-helper cells (Th1), their production of IFN- $\gamma$ and TNF- $\alpha$, maturation and antigen-presenting function of dendritic cells and macrophages, and the production of proinflammatory cytokines and chemokines (IL-1 $\alpha$, IL-1 $\beta$, IL-6, IL-12, TNF- $\alpha$, CCL2, CCL5, IL-8). Due to the formation of a CCL2 antagonist, MSCs can also inhibit the migration of proinflammatory CCR2 + monocytes to the 
center of inflammation. In this case, MSCs stimulate the formation of type $2 \mathrm{~T}$-helper cells (Th2) and regulatory populations of $\mathrm{T}$ and $\mathrm{B}$ lymphocytes (Treg and Breg, respectively). These properties of MSCs determine their anti-inflammatory activity, which is potentiated in the "inflammatory" microenvironment, in particular, in the presence of IFN- $\gamma$, TNF- $\alpha$, which allows us to consider MSCs not as an immunosuppressant but as an immune-regulating cell population (Lyadova et al., 2016; Jiang and $\mathrm{Xu}, 2020$ ).

The anti-inflammatory activity of MSCs is confirmed by numerous in vivo studies. In experimental sepsis models, intravenous administration of MSCs obtained from various sources (bone marrow, adipose tissue, cord blood) reduced the level of proinflammatory cytokines TNF- $\alpha$ and IL-6, stimulated the production of IL-10 by macrophages, and reduced the severity of sepsis and caused animal mortality (Németh et al., 2002; Ahn et al., 2020). In the mouse model of emphysema, human bone marrow MSCs therapy and application of MSCs-conditioned medium had a significant cytoprotective effect when used at the early stages (Kennelly et al., 2016). These protective effects were due to substantial anti-inflammatory, antifibrotic, and antiapoptotic properties.

At the same time, studies on animals indicate the possibility of low effectiveness and side effects when MSCs are administered. Thus, in the pig model of sepsis, the introduction of MSCs did not reveal any significant positive effects (Horak et al., 2020) and did not reduce hemodynamic changes and the development of multiple organ failure caused by sepsis. In mice with a pronounced inflammatory process in the lung tissue caused by $M$. tuberculosis infection, MSCs did not provide an effective reduction in the inflammatory process (Nenasheva et al., 2017).

Side effects of MSCs are primarily associated with their procoagulant activity and the risk of thrombosis due to the production of the tissue factor and possibly activation of the complement system (discussed in detail in the review of Coppin et al. (2017). The tissue factor (TF) and other proteins involved in the blood coagulation system are revealed in extracellular secreted vesicles obtained from MSCs. It was also found that some MSCs and their vesicles contained annexin $\mathrm{V}$, which means the presence of phosphatidylserine on their surfaces, which increases the formation of a blood clot (Silachev et al., 2019). In case of systemic administration to healthy animals, MSCs can cause blockage of the capillary bed of lung tissue and, in addition to reduction in the production of proinflammatory cytokines (IL-6, IL-12, IFN- $\gamma$ ), induce an increase in local chemokine levels (CCL3, CCL4, RANTES) (Nenasheva et al., 2017).

Another important point is that the decrease in the inflammatory response caused by MSCs is accompanied by inhibition of immune responses which are responsible for the body's protective functions, in par- ticular, for the development of antiviral and antibacterial responses (inhibition of the formation and activity of Th1, NK cells). In this regard, the application of MSCs in conditions of an infectious pathology is impossible without a clear definition of immunological criteria for the reasonability of their use ("windows of opportunities").

The results from clinical studies of MSCs are also very contradictory (Gomez-Salazar et al., 2010) and often do not achieve their goals. The use of MSCs to mitigate the severe "graft-versus-host" reaction that is refractory to steroids is the most developed method. However, no significant clinical effect of the MSCsbased Prochymal ${ }^{\mathrm{TM}}$ drug was obtained in phase III clinical trials. This drug, however, was allowed in some countries to treat this pathology in children, because this group of patients demonstrated a significant effect compared with the placebo group. Studies to assess the potential of MSCs for the treatment of diabetes, liver, kidney, and lung pathologies; the cardiovascular system; the musculoskeletal system; and inflammatory, neurological, and autoimmune diseases have been carried out (Squillaro et al., 2016). Acute respiratory distress syndrome (ARDS) is also considered in the context of possible treatment by MSCs (Han et al., 2019). However, ARDS is a heterogeneous syndrome; based on the analysis of clinical research data, it is concluded that there are at least two types of ARDS, of which only one was characterized by higher levels of inflammatory biomarkers in plasma (Calfee et al., 2014). Perhaps, MSCs therapy for such patients with hyperinflammatory ARDS type will be most effective.

In experimental models of viral ARDS, the effect of MSCs was also ambiguous. The lung damage caused by the H1N1 flu virus in mice was not suppressed by the administration of MSCs (Gotts et al., 2014). However, an introduction of MSCs significantly weakened the damage of the alveolar-capillary barrier in H5N1-infected mice, which have a high level of inflammation, and increased the chance of survival of the animals (Chan et al., 2016). In clinical studies, the introduction of allogeneic MSCs from adipose tissue in patients with ARDS did not have a positive clinical effect and did not cause any statistically significant changes in blood's biochemical parameters, in particular, in the levels of IL-8 and IL-6 (Zheng et al., 2016).

Although there are no results from serious clinical studies of long-term effects and the risk/benefit ratio of intravenous MSCs administration, according to available information, the number of short-term side effects and complications is low. Among short-term complications, reports of the risk of thrombosis caused by therapy are noteworthy (Tatsumi et al., 2017). In the situation of administration of lipopolysaccharide to healthy people, the preadministration of high-dose of MSCs had both proinflammatory (increased 
release of IL-8 and nucleosomes) and anti-inflammatory (increased release of IL-10 and TGF- $\beta$ ) effects, as well as increased activation of the coagulation system and, decreased blood fibrinolytic activity (Perlee et al., 2018).

There is no answer yet to the most important question about the possible induction of tumor growth by transplanted MSCs due to modification of the tumor stroma (Direkze et al., 2004).

Thus, there is very little reliable data on the mechanism of action of MSCs in the course of systemic administration to humans as well as on the distribution of cells in the body and the long-term consequences of such administration; the data from model experiments are contradictory both concerning the specific action of MSCs and their safety.

However, soon after the beginning of the SARSCov-2 pandemic, clinical trials were initiated using MSCs to treat severe manifestations of this infection. Most of these studies started in the Chinese People's Republic, but there were reports from other countries soon after that, and their number is constantly growing (more than by two times a week).

In this regard, we attempted to evaluate the scale of studies of the possible therapeutic effect of MSCs in human COVID-19 based on the information from the international database of clinical trials ClinicalTrials.gov (https://clinicaltrials.gov) (Table 1 in the Appendix on the website of the journal Ontogenez). At the time of writing of this paper, 29 clinical trials of biomedical cell products (BMCP) (including one product that was canceled) for the treatment of COVID-19 complications, as well as one study of cell vesicles' application, have been officially registered. Eighteen of them meet the criteria for clinical trials, while others can be described by the term "Clinical tests." Also, according to available data, several clinical trials have been conducted with registration only in the Chinese People Republic (Golchin et al., 2020). The umbilical cord cells are most often used as a source for the cell product, which is probably due to the availability and/or properties of the biomaterial.

Possibly, the lack of serious evidence on the effectiveness of products based on living human cells in the COVID-19 condition did not allow them to be mentioned in Coronavirus Disease 2019 (COVID-19) Treatment Guidelines of the US National Institute of Health (released on April 21, 2020) as potentially promising. It is noted in the guidelines that no one drug is currently safe and effective for the treatment of COVID-19; empirically selected and currently used treatments have a small restricting effect on the development of symptoms of pneumonia, including fatal pulmonary failure. The guidelines note that there are no FDA-approved drugs specifically designed to treat patients with COVID-19; the data are insufficient to recommend the use of antiviral or immunomodulatory therapy for patients with COVID-19 (Coronavi- rus Disease 2019 (COVID-19) Treatment Guidelines). However, if clinical studies show an acceptable risk/benefit ratio for the application of MSCs, countries where such studies have been conducted can expect their introduction into medical practice, probably not earlier than within 1 year.

In Russia, however, the current legislation in the field of BMCP does not provide any mechanisms for accelerated or simplified registration of BMCP (in contrast to many foreign countries, where Fast Track or conditional registration mechanisms are stipulated for such cases) or a mechanism for accelerated obtaining of permission to conduct clinical trials. Even if the special mechanism for BMCP will be developed and approved, the conducting of evidence-based preclinical studies and the production of samples of BMCP for clinical trials at properly licensed facilities are the critical issues.

It is extremely important, on the one hand, not to miss the chance for the medicine to find a new effective tool for the treatment of diseases incurable by conventional methods (or conditions with a high fatality), and, on the other hand, to prevent the mass usage of $\mathrm{BMCP}$ with unclear risk/benefit ratio. At first, it is necessary to conduct an experimental verification of a specific effect of MSCs and the risks of their usage in the COVID-19 condition in sufficient volume and, in parallel, to create a mechanism for accelerated but reasonable admission of BMCP into practice. Otherwise, the detection of serious side effects may occur later, and $\mathrm{BMCP}$ will be discredited completely.

\section{FUNDING}

The research was performed within the framework of a State Assignment of Koltzov Institute of Developmental Biology of Russian Academy of Sciences, no. 0108-20190004. 2020.

\section{COMPLIANCE WITH ETHICAL STANDARDS}

The authors declare that they have no conflict of interest. This paper does not contain any studies involving animals or human participants performed by the authors.

\section{REFERENCES}

Ahn, S.Y., Maeng, Y.-S., Kim, Y.R., et al., In vivo monitoring of dynamic interaction between neutrophil and human umbilical cord blood-derived mesenchymal stem cell in mouse liver during sepsis, Stem Cell Res. Ther., 2020, vol. 11, no. 1, pp. 44-59.

Calfee, C.S., Delucchi, K., Parsons, P.E., et al., Subphenotypes in acute respiratory distress syndrome: latent class analysis of data from two randomized controlled trials, Lancet Respir. Med., 2014, vol. 2, no. 8, pp. 611-620.

Chan, M.C., Kuok, D.I., Leung, C.Y., et al., Human mesenchymal stromal cells reduce influenza A H5N1-associated acute lung injury in vitro and in vivo, Proc. Natl. 
Acad. Sci. U. S. A., 2016, vol. 113, no. 13, pp. 36213626.

Coppin, L., Sokal, E., and Stephenne, X., Thrombogenic risk induced by intravascular mesenchymal stem cell therapy: current status and future perspectives, Cells, 2019, vol. 8, no. 10, p. 1160.

Coronavirus Disease 2019 (COVID-19) Treatment Guidelines. https://www.covid19treatmentguidelines.nih.gov/.

Direkze, N.C., Hodivala-Dilke, K., Jeffery, R., et al., Bone marrow contribution to tumor-associated myofibroblasts and fibroblasts, Cancer Res., 2004, vol. 64, no. 23, pp. 8492-8495.

Golchin, A., Seyedjafari, E., and Ardeshirylajimi, A., Mesenchymal stem cell therapy for COVID-19: present or future, Stem Cell Rev. Rep., 2020, pp. 1-7.

Gomez-Salazar, M., Gonzalez-Galofre, Z.N., Casamitjana, J., et al., Five decades later, are mesenchymal stem cells still relevant?, Front. Bioeng. Biotechnol., 2020, vol. 8 , no. 148 , pp. $1-13$.

Gotts, J.E., Abbott, J., and Matthay, M.A., Influenza causes prolonged disruption of the alveolar-capillary barrier in mice unresponsive to mesenchymal stem cell therapy, Am. J. Physiol. Lung Cell Mol. Physiol., 2014, vol. 307, no. 5, pp. L395-L406.

Han, J., Li, Y., and Li, Y., Strategies to enhance mesenchymal stem cell-based therapies for acute respiratory distress syndrome, Stem Cells Int., 2019, no. 5432134, pp. 1-12. https://clinicaltrials.gov.

Horak, J., Nalos, L., Martinkova, V., et al., Evaluation of mesenchymal stem cell therapy for sepsis: a randomized controlled porcine study, Front. Immunol., 2020, vol. 11, no. 126 , pp. 1-13. https://clinicaltrials.gov.

Jiang, W. and Xu, J., Immune modulation by mesenchymal stem cells, Cell Prolif., 2020, vol. 53. e12712.

Kennelly, H., Mahon, B., and English, K., Human mesenchymal stromal cells exert HGF dependent cytoprotective effects in a human relevant pre-clinical model of COPD, Sci. Rep., 2016, vol. 6, no. 38207, pp. 1-11.
Lyadova, I.V., Sosunova, E., Nikolaev, A.V., et al., Mesenchymal stem cells and myeloid derived suppressor cells: common traits in immune regulation, J. Immunol. Res., 2016, vol. 2016, 7121580.

Németh, K., Leelahavanichkul, A., Yuen, P.S., et al., Bone marrow stromal cells attenuate sepsis via prostaglandin $\mathrm{E}(2)$-dependent reprogramming of host macrophages to increase their interleukin-10 production, Nat. Med., 2009, vol. 15 , no. 1 , pp. 42-49.

Nenasheva, T., Nikolaev, A., Diykanov, D., et al., The introduction of mesenchymal stromal cells induces different immunological responses in the lungs of healthy and $\mathrm{m}$. tuberculosis infected mice, PLoS One, 2017, vol. 12, no. 6. e0178983.

Perlee, D., van Vught, L.A., Scicluna, B.P., et al., Intravenous infusion of human adipose mesenchymal stem cells modifies the host response to lipopolysaccharide in humans: a randomized, single-blind, parallel group, placebo controlled trial, Stem Cells, 2018, vol. 36, no. 11 , pp. $1778-1788$.

Silachev, D.N., Goryunov, K.V., Shpilyuk, M.A., et al., Effect of MSCs and MSC-derived extracellular vesicles on human blood coagulation, Cells, 2019, vol. 8, no. 258 , pp. $1-23$.

Squillaro, T., Peluso, G., Galderisi, U., et al., Clinical trials with mesenchymal stem cells: an update, Cell Transplant., 2016, vol. 25, no. 5, pp. 829-848.

Tatsumi, K., Ohashi, K., Matsubara, Y., et al., Tissue factor triggers procoagulation in transplanted mesenchymal stem cells leading to thromboembolism, Biochem. Biophys. Res. Commun., 2013, vol. 431, no. 2, pp. 203-209.

Zheng, G., Huang, L., Tong, H., et al., Treatment of acute respiratory distress syndrome with allogeneic adiposederived mesenchymal stem cells: a randomized, placebo-controlled pilot study, Respir. Res., 2014, vol. 15, no. 39, pp. 1-10.

Translated by A. Ermakov 